\title{
Juegos tradicionales y nuevas tecnologías: Continuidades y apropiaciones
}

\author{
Carolina Duek \\ Noelia Enriz
}

\section{Juegos tradicionales y nuevas tecnologías: Continuidades y apropiaciones}

Resumen: El aumento de los dispositivos tecnológicos disponibles relacionados a los jóvenes y a los niños en la Argentina generó el resurgimiento de un debate entre los padres, docentes e investigadores: ¿cuáles son las transformaciones que los juegos infantiles sufren a causa de la presencia de las nuevas tecnologías de la información y comunicación? ¿Cómo es posible clasificar y analizar el nuevo universo que se abre? ¿Existen continuidades o podemos hablar solamente de transformaciones y cambios? El objetivo del presente artículo es la identificación de la relación entre los juegos "tradicionales" y los juegos que están disponibles en diferentes soportes tecnológicos y materiales. La hipótesis que sostendremos afirma que existen muchas continuidades entre los juegos tradicionales y los nuevos juegos. Esta hipótesis será trabajada con datos obtenidos de nuestra investigación, la realización de un análisis complejo sobre las relaciones entre los juegos contemporáneos y los niños que juegan con ellos.

Palabras Clave: Infancia. Juegos. Transformaciones.

\section{Traditional games and new technologies: Continuities and appropriations}

Abstract: The increase in the availability of technological devices related to children and people in Argentina has generated the reappearance of a debate among parents, professors and researchers: Which are the transformations that children's games suffer as a result of the presence of new technologies of information and communication? How is it possible to classify and analyze the new universe that opens with it? Are there continuities or we can only detect changes and transformations? The objective of this paper is to identify the relationship between "traditional games" and those that are available in technological and material supports. The hypothesis that we sustain claims that there are many continuities between traditional games and those new ones that are organized in and between new technologies. This hypothesis will be developed with information from our research and through the analysis of the complex relationship between contemporary games and children that play with them.

Key words: Childhood. Games. Transformations. 


\section{Introducción}

Los juegos y los niños tienen una relación que puede ser identificada a lo largo de toda la historia moderna. La característica distintiva de este vínculo en el contexto contemporáneo está signada por la aparición de la industria de producción de juegos y juguetes en tanto actores clave de los juegos infantiles y de las nuevas tecnologías. Es por ello que nos proponemos componer una aproximación integral sobre las diferentes relaciones que identificamos entre los niños, los medios de comunicación y las nuevas tecnologías de la información y la comunicación. En este contexto, es necesario identificar los diferentes agentes que intervienen en los juegos pero, también, en la producción de representaciones hegemónicas de las prácticas culturales contemporáneas.

Los juegos infantiles, en tanto que prácticas culturales, nos brindan una puerta de entrada al análisis de la vida cotidiana de los niños, a la constitución de la cultura pero, también, a los significados que los propios jugadores le otorgan a sus juegos. Nuestras investigaciones nos han permitido articular dos contextos diferentes: por un lado, los análisis que habitan en las grandes ciudades y, por otro, las investigaciones sobre los niños que forman parte de las poblaciones originarias de la Argentina.

Duek (2012; 2013) analizó la relación existente entre los niños, los juegos y los medios de comunicación con un abordaje inscripto en las Ciencias de la Comunicación. Sus investigaciones presentan una mirada sobre la imbricación de los medios en la vida cotidiana de los niños en diferentes espacios y formas de vinculación. Los roles de los adultos, las representaciones sociales y mediáticas y las relaciones complejas entre el ecosistema comunicativo y los sujetos en tanto claves para un análisis interdisciplinario.

Enriz $(2011$; 2012) realizó sus investigaciones con los Mbyá Guaraní en la provincia de Misiones, Argentina. En sus trabajos, la antropóloga mostró las maneras de organización de la vida familiar, grupal y geográfica entre los sujetos. Una de las dimensiones centrales en su investigación es el rol de los juegos en la vida y dinámicas de los Mbyás Guaraníes. Ella entendía los juegos en el sentido que los propios sujetos de la investigación le asignaban con una perspectiva etnográfica centrada en la voz y en las prácticas de los informantes.

El encuentro entre estos dos caminos de investigación comienza por los marcos teóricos con los cuales analizamos los juegos en tanto que prácticas culturales significativas. Huizinga (1939) y Caillois (1964) son probablemente las referencias más importantes para cualquier abordaje sobre los juegos y las relaciones con la cultura y los contextos sociales. Pero también Sutton Smith (1959, 1971 y 1975), Brougère (1998 y 2013), Manson y De la Ville (2013), Nunes (2013), entre tantos otros, son referencias claves para nuestras investigaciones más allá de los diferentes campos de inserción de los objetos de estudio.

La continuidad de nuestro « encuentro » puede encontrarse, también, en proyectos de investigación conjuntos ${ }^{1}$ y en la creación de una revista científica sobre juegos y cultura

${ }^{1}$ Los proyectos de investigación que han permitido el análisis que aquí presentamos son el PICT (ANPCyT) 2010-1913 (2011-2012) y el PIP (CONICET) 2014-2016. Ambos proyectos abordan problemáticas vinculadas al juego, a las consideraciones de niños y niñas sobre sus prácticas lúdicas y a los entornos comunicativos que rodean a las infancias contemporáneas en las grandes ciudades. 
que se llama « Lúdicamente ${ }^{2}$ ». La articulación entre la antropología y las ciencias de la comunicación nos ha brindado la posibilidad de construir una mirada, al mismo tiempo, compleja y original sobre los juegos, los niños y la cultura contemporánea.

El objetivo del presente artículo es presentar un análisis sobre los juegos y las nuevas tecnologías a través de una hipótesis, por lo menos, polémica. Ella sostiene que los juegos infantiles contemporáneos tienen muchos más elementos y características de continuidad que de diferencia con los juegos "tradicionales" (aunque sean presentados en soportes variados y aparentemente originales). La hipótesis nos permitirá analizar algunos juegos contemporáneos en múltiples dimensiones y apropiaciones.

Las preguntas que organizan el artículo son: ¿Cómo podemos identificar las tensiones y las transformaciones entre los juegos « tradicionales » y los juegos que están ubicados en dispositivos y gadgets tecnológicos? ¿Qué dinámicas lúdicas son pasibles de ser identificadas en las estructuras de los juegos contemporáneos? ¿Cómo es posible articular los diferentes tipos de juego según los propios testimonios de los niños y niñas entrevistadas?

En lo respectivo a la metodología, es necesario hacer algunas aclaraciones. Este trabajo es una articulación de una gran diversidad de investigaciones que hemos llevado adelante a lo largo de los años. Es por ello que presentaremos una combinación de perspectivas y de decisiones metodológicas que se articulan sostenidamente a lo largo de este trabajo. El marco general a nivel metodológico son las investigaciones cualitativas y utilizamos el entrevista antropológica en la realización de las entrevistas a informantes clave de los grupos en análisis. La combinación del análisis del discurso con las herramientas propias de la etnografía, las entrevistas en profundidad y algunas formas de análisis vinculadas a la sociosemiótica operan como un amplio arco sobre el cual se asientan nuestras investigaciones actuales.

Para trabajar nuestra hipótesis hemos elegido dos dimensiones analíticas sobre las cuales organizar el artículo. Estas dimensiones van a funcionar como grandes ejemplos sintetizadores de una reflexión que, claramente, los excede. Hemos seleccionado estas dimensiones porque consideramos que condensan en su mayoria las apropiaciones y las consideraciones que vinculan a los juegos "tradicionales" con los contemporáneos que están vinculados con nuevos soportes y tecnologías. A la primera de las dimensiones la llamamos «el dominó como juego residual » y la segunda aborda la relación entre las armas, los soldados y la historia de los juguetes. Estas dimensiones nos permitirán abrir espacios conceptuales y teóricos para presentar un análisis sobre la relación entre los juegos, los niños, las nuevas tecnologías y la cultura contemporánea. No se trata de hacer un análisis generalizador sino de buscar, en el sentido de Hoggart (1990) las invariantes culturales que se nos han presentado a lo largo de la investigación. El dominó y los juegos "de guerra" son dos grandes ejes sobre los cuales proyectaremos nuestra hipótesis con el objetivo de contribuir a la reflexión sobre los juegos, los niños y la cultura contemporánea.

\footnotetext{
${ }^{2}$ Lúdicamente (ISSN 2250-723X) es una revista que ha publicado 6 números desde el año 2012 y que se encuentra indexada en Latindex, DialNet, e-revistas, DOAJ, Sherpa Romeo y Qualis, por mencionar algunas de todas las existentes. La publicación tiene como objetivo la apertura de un espacio de publicación científica de investigaciones sobre juego desde una perspectiva multidisciplinaria.
} 


\section{El dominó como juego residual}

El dominó pertenece al conjunto de los «juegos tradicionales »(GLANZER, 2000). La invención (o los primeros registros existentes) de este juego puede ser ubicada en el pasado y esto significa, para nuestro análisis, que nuestro objeto de estudio en esta primera dimensión nos va a permitir la construcción de una reflexión sobre los cambios de la práctica del dominó, las diferentes formas de jugar y las dinámicas y los objetos destinados para el juego. El dominó, en tanto juego tradicional, nos brinda una entrada privilegiada al universo lúdico en el cual ubicamos nuestro análisis.

La presencia del dominó en la historia de los juegos es tan importante que encontramos registros de su existencia como «pasa-tiempo » en momentos de conflictos y de guerras. La noción de distracción estaba presente en los primeros documentos encontrados sobre el dominó en la región de Cuba y en América central. El dominó demanda la combinación entre dos números e imágenes iguales: hay que conectar una imagen detrás de la otra partiendo del principio según el cual cada pieza debe ser seguida por su igual. No es éste un juego difícil de comprender o de jugar: la simplicidad del juego es, probablemente, la explicación de su lugar en la historia de los juegos. Esto no significa que consideremos al dominó como un juego fácil, pero sí es cierto que no es necesario tener competencias específicas para comprender la dinámica básica del juego (que sí son necesarias para la construcción de estrategias y de combinaciones virtuosas para imponerse al o a los rivales).

Encontramos diferentes variantes para jugar al dominó en América Latina y Central (para mayores precisiones consultar Rodriguez Tapanés y Hernández de Lara, 2008): múltiples combinaciones de cantidades de números, piezas e imágenes... pero siempre con la misma dinámica de juego. La conexión y la construcción de una línea de juego con el objetivo de descartar las piezas lo más rápido posible era el vector común de todas las versiones. $\mathrm{El}$ « doble 9 » o el « doble 12 »como las piezas más altas del juego pueden ser encontradas en diferentes versiones y disposiciones lúdicas (para ampliar, consultar Plath, 1986). Rossi Cardoso (2001) encontró que las diferentes formas de jugar están conectadas con las tradiciones familiares y, también, regionales. En otras palabras, es necesario conocer no solamente la historia de los juegos sino, también, los espacios y los significados que los juegos (y sus variables) tienen para los propios jugadores en un momento de su historia social, cultural y educativa (Pelegrinelli, 2010).

Todos estos elementos no han permitido arribar a una conclusión intermedia: el dominó es, en el curso de la historia, un juego residual, tal como define Williams (1988) la dimensión en la cultura. Es decir, el dominó es un juego que tiene una dimensión de su propia historia (de su pasasdo) que está presente en los tiempos contemporáneos de manera «actualizada». Los elementos residuales de la cultura son, para Williams, la manera a través de la cual el pasado se resignifica en el presente y la forma en la cual los sujetos, en tanto que sujetos sociales, convocan sus pasados en sus vidas presentes.

La cultura, considerada como un producto histórico, puede ser analizada solamente en el curso histórico que le permite desarrollarse de una manera específica en un contexto preciso. La investigación sobre el dominó nos ha brindado ciertos resultados sorprendentes: seguimos su curso histórico pero, también, las apropiaciones que diferentes agentes sociales han hecho de este juego. Es por ello que este juego está presente en diferentes dominios de la vida cotidiana de maneras variadas. En los próximos párrafos presentaremos algunos usos y apropiaciones identificados del 
dominó para exponer las dimensiones residuales y también las variables que intervienen en cada configuración de la práctica del dominó en tanto que juego. Los ejemplos, en cada caso, son ilustrativos y reponen lo significativo de su presencia ya no sólo como juego sino como práctica cultural significativa.

En la actualidad, el dominó se encuentra articulado con diferentes dimensiones de la cultura que explican las apropiaciones y los usos del juego. En primer lugar, una de las más frecuentes apropiaciones proviene del mercado de juegos y juguetes. Múltiples variantes, temas y personajes aparecen en las pequeñas fichas de dominó como una manera de "actualizar" ya no el juego y su dinámica sino la superficie y la presentación de las fichas. Barbies ${ }^{3}$, Ben $_{10}{ }^{4}$ y cualquier personaje de moda pasan rápidamente a ocupar espacios en juegos, juguetes y útiles escolares. Cada una de las apropiaciones que analizaremos se organiza en torno a diferentes estrategias que no modifican, finalmente, la dinámica del juego. Lo que sí nos permiten ver son los contextos y los significados que se le imprime a cada una de las apropiaciones.

El dominó no es la excepción sino que sigue el mismo curso que otros juegos "tradicionales" en tensión y vinculación con el mercado de bienes (SCHEINES, 1985). No se trata de desplazar el juego en sí mismo sino de imprimirle características contemporáneas que le permiten ocupar espacios de deseo entre los niños que siguen ya no a los juegos sino a los personajes. Un niño-promedio al que le gusta Ben10 no sólo ve el programa en televisión, sino que puede llegar a demandar todos los productos disponibles vinculados al personaje y puede desear jugar a los juegos y que pida específicamente aquellos que tengan "algo" que lo relacione con ese personaje.

Es por ello que la apropiación del dominó por parte del mercado es tan compleja: no se propone una modificación del juego sino que avanza en una metamorfosis de la superficie y de la estética de presentación para llamar la atención de los niños ya no como jugadores sino como seguidores de una marca. El personaje, devenido en marca, opera como un gran organizador de los juegos infantiles desde la perspectiva del mercado. No estamos sosteniendo que esto es, efectivamente, así sino que existe una serie de mecanismos que contribuyen a posicionar los productos relacionados con los personajes de los programas de televisión en una trama infinita de oferta, demanda y consumo. En esta primera apropiación, el dominó es una excusa, una manera de usar juegos desde una perspectiva residual para transformarlos no sólo en objetos actuales sino en "deseables" por la figura convocante (MOLINARI y CANTORA, 2012) que apunta a conquistar la atención de los más chicos. Al reemplazar un número por la imagen de un personaje, el dominó mantiene su lógica de juego pero se reconvierte hacia el fetichismo de y sobre los personajes convocados: la igualación de dos princesas o de dos personajes desplaza la asociación de puntos y la transforma en una excusa para seguir vinculados con las marcas, los personajes y la cadena infinita de consumo que se propone desde ellos.

${ }^{3}$ La globalmente famosa muñeca de Mattel que, a lo largo de su historia se transformó en un ícono no sólo de los juegos y juguetes sino de la expansión de una marca a escala global. No sólo en su página web se presentan las muñecas y accesorios sino las películas, juegos vinculados y todas las posibilidades de vinculación de los más chicos con la marca (http://www.barbie.com/es-lam).

${ }^{4}$ Uno de los programas de Cartoon Network más vistos en América Latina en los últimos años y una de las marcas más importantes del mercado de juegos, juguetes y merchandising asociado (mochilas, indumentaria, accesorios para la escuela, entre tantos otros (http://www.cartoonnetwork.com/games/ben10/index.html). 
Una segunda apropiación es la de las instituciones educativas. En muchos colegios, el dominó es utilizado como un camino lúdico de aprendizaje de las matemáticas y de los números y sus asociaciones de manera específica. Muchas veces en los colegios se construyen juegos con la colaboración de los alumnos con diferentes materiales y combinaciones. Lo que queda invariable es, por un lado, la dinámica de juego y, por otro, la estructura más tradicional que suele ser reproducida con los números y con combinaciones que terminan en el seis/seis (una de las variantes más extendidas globalmente desde sus inicios como juego). Muchos diseños curriculares proponen la utilización de los juegos como forma de enseñanza y de aprendizaje. El juego, transformado en estrategia, busca a la vez convocar el interés de los niños y, complementariamente, transmitir conocimientos matemáticos y de geometría por medio de la asociación de formas y de puntos que se suman para componer números. La apropiación por parte de las escuelas de los juegos como medio para la enseñanza puede desplazar el placer del juego en sí mismo porque se lo asocia como un camino necesario para llegar a un objetivo curricular. Es decir, el juego se transforma en un soporte educativo, en una excusa, y se resignifica en otro contexto (el curricular/escolar). "Juguemos al dominó" deja de ser un juego y se transforma, metafóricamente, en una hoja de un manual escolar que debe ser no sólo seguida al pie de la letra sino comprendida e incorporada como contenido obligatorio.

En ambas apropiaciones del dominó (expuesta aquí de modo ilustrativo y retomando algunas de las más frecuentes), la del mercado y la escolar, encontramos la utilización de rasgos que favorecen a los intereses y objetivos de quienes diseñan y organizan los juegos y los espacios de desarrollo. No es nuestro objetivo plantear estas apropiaciones como "desvíos", sino marcar las formas en las que el contexto de juego modifica de manera central las dinámicas y las finalidades de las experiencias lúdicas. Es por ello que creemos que no es posible analizar los juegos contemporáneos sin dar cuenta del contexto mayor en el que se ubican, presentan y apropian (en este caso por pare de los jugadores aunque aquí no hemos profundizado esta dimensión. Para ampliarla consultar Duek, 2014 y Enriz, 2011).

El dominó, probablemente uno de los juegos más importantes en la historia por su extenso recorrido a través de diferentes contextos y características, nos brindó una puerta de entrada a la relación entre los juegos y diferentes agentes que participan de manera decisiva en la cotidianeidad infantil. El mercado de bienes, los programas de televisión, los juegos, los juguetes, los videojuegos pero también la escuela, los docentes y los objetivos pedagógico-didácticos, se articulan en un mismo terreno con diferentes objetivos. El significado de un juego se construye, también, en función de sus jugadores y los contextos.

\section{Jugar a matar: un campo consolidado y en expansión}

Otro de los espacios que nos permite analizar los juegos en la actualidad es el que podríamos denominar "de guerra". Incluimos aquí desde los soldaditos de plomo hasta sus versiones plásticas, las armas, los arcos y flechas, los videojuegos que se organizan en torno a luchas armadas, sólo por mencionar algunos objetos y prácticas aquí incluidas. Analizar los juegos vinculados a las armas y a la guerra nos ubica en un espacio de juegos tradicionales diferente al del dominó. Como sostienen Manson y De 
La Ville (2013), los materiales utilizados en la producción de los juguetes en siglos pasados son tan diferentes que los juegos que permitían también lo eran (por no mencionar los citados casos de contaminación y de intoxicación hasta la muerte sobre los que trabajan ambos autores).

La cantidad de objetos disponibles, la durabilidad y su calidad configuraban de diferentes maneras los juegos. Pero también, como explica Brougère (2008 y 2013), estos juegos están muy asociados con el género: los niños varones y los juegos de guerra y de soldados han sido asociados desde hace muchos siglos. Benjamin (1990) identifica a los soldaditos de plomo con las texturas de la infancia y con la frialdad que tenían, al tacto, esas miniaturas del mundo adulto que aparecían una y otra vez como explicación de lo que ocurría a su alrededor. La fuerza, la rigidez y la imposibilidad de alterar los pequeños muñecos se explicaba en relación al contexto en el que se utilizaban y en el que se les imprimía un significado específico. En la actualidad, queda en los coleccionistas que intentan reponer, a través de la acumulación organizada de objetos, los residuos de los sentidos de ese pasado (en este caso la noción de residual es diferente porque el coleccionista resignifca esos objetos reponiendo el significado del pasado sin reactualizarlo en el presente, en el sentido que define Williams, 1998 lo arcaico de la cultura).

El lugar que ocupan, en la actualidad, los juegos de guerra o los elementos que pertenecen a dicho campo se han desplazado hacia diversos soportes. Por un lado, encontramos cientos de soldaditos de plástico que se venden en jugueterías pero también muchas veces en el mercado informal por muy poco dinero. El significado actual de la figura del "soldado" ya no es representativa del mundo adulto. En la Argentina, no existe una construcción fuerte de la relación entre los soldados-la defensa del territorio-la patria. De hecho, el último conflicto armado en el que participó fue la llamada "Guerra de las Malvinas" en 1982, un conflicto organizado por medio de la reivindicación de la soberanía de las Islas por parte de un gobierno dictatorial (GUBER, 2004). La falta de legitimidad del conflicto y su excepcionalidad en la historia argentina reciente, ubica la noción de "guerra" no sólo lejos de la cotidianeidad infantil sino que, para los que recuerdan dicho conflicto, opera como un pasado vergonzante que no se reactualiza en el presente. El lugar de los juegos de guerra en la Argentina se vincula, entonces, con el rol de algunos juegos de mesa, los de imitación (en el sentido del mimicry de Caillois, 1994) y los videojuegos. Matar, sin importar a quien, es una clave que encontramos de manera recurrente en juegos electrónicos. Desde el Wolfenstein creado en 1992, hemos presenciado un crecimiento exponencial ya no sólo de la industria sino de la presencia de las armas y de las escenas de guerra y combate en muchos de los juegos electrónicos más vendidos y más usados. Uno de los casos emblemáticos es el del GTA ${ }^{5}$ (Gran Theft Auto) que, desde 1997 y con múltiple versiones, puso en escena un nivel inédito de violencia sobre los monitores de las computadoras. La clave en el juego era no sólo el asesinato sino la ejecución de diferentes personajes en función de "misiones". El peso lúdico estaba puesto en el asesinato y en la huida permanente de diferentes ciudades y espacios urbanos con la ayuda de un vehículo sobre el cual se montaba el personaje principal. No creemos que, como muchos estudios sostienen, la violencia en la pantalla "refleje" ni "ilustre" la

La página oficial en la que se encuentran las múltiples versiones del juego es: http://www.rockstargames.com/grandtheftauto/. 
violencia social (cfr. GARCÍA GALERA, et al., 2000). Tampoco creemos que la violencia en los juegos contribuya a un aumento de la violencia social. Lo que sí queremos dejar aquí en claro es el aumento exponencial de juegos de guerra que utilizan como herramienta principal a la violencia (con la ayuda de armas y de otros implementos "mortales") y la sofisticación de las maneras de matar y eliminar diferentes adversarios. Los elementos que quedan desplazados son aquellos vinculados a la estrategia del enfrentamiento y al diseño de alternativas creativas para vencer a un "enemigo". Los personajes de este juego nunca están del lado de la ley. En las últimas versiones encontramos edulcorado el lugar de "delincuente" que se desplazó hacia "un marginado" o "un ser maltratado por la sociedad". De este modo, los diseñadores del GTA responden a las críticas que los acusaban de apología de la delincuencia y de la criminalidad.

La guerra, el enfrentamiento y el crimen aparecen, finalmente, como una escena frecuente en los juegos de consolas y electrónicos pero es crecientemente desplazada de los juguetes y juegos de mesa. Hemos analizado la gran presencia de pistolas y armas como accesorios de las consolas de juego (Xbox, Wii, PlayStation) y, si bien siguen existiendo las armas en venta en jugueterías, el peso significativo en lo respectivo a los juegos lo encontramos más en los videojuegos que en la oferta disponible en jugueterías. Esto tiene una explicación: la mejora de la calidad de las pantallas, de los diseños y de los gráficos ha permitido un mayor margen de maniobra respecto de lo que los personajes hacen, deben y pueden hacer mientras que los objetos lúdicos en tanto auxiliares dependen casi exclusivamente de las decisiones de los sujetos. Una vez más encontramos que el mercado se ocupa de perfeccionar sus propuestas y de limitar las acciones de los jugadores para orientarlas en su propia dirección y conveniencia (Levis, 2003). Este proceso lo hemos identificado, también, en la presencia de instrucciones cada vez más ajustadas y de acciones más delimitadas en los juegos de mesa: hay un menor margen de intervención de los jugadores y una mayor presencia de las reglas y de dinámicas pre-establecidas de juego.

Otra dimensión que identificamos en el trabajo etnográfico (y que puede considerarse la contracara de este proceso) se vincula con la venta de "souvenirs" o pequeños recuerdos de viaje en la región de Misiones por parte de la comunidad Mbyá Guaraní y la comunidad Wichí en el Gran Chaco (para ampliar y precisar la etnografía, ver Enriz, 2011). Si bien existen múltiples casos idénticos, este que presentamos ha sido identificado en un trabajo etnográfico. Uno de los objetos que vendían estas comunidades a los turistas eran arcos y flechas que se realizaban de manera artesanal con maderas, fibras vegetales y demás elementos accesibles en el monte. La clave que identificó Enriz radicaba en el significado que dichos elementos tenían en la historia de quienes los producían y la manera en la que éstos eran adquiridos como recuerdos.

Para los turistas, el arco y la flecha representaban la guerra y el enfrentamiento con otros, es decir, simbolizaban un universo de tensión y de combate. Pudieron registrarse escenas en que los niños que compraban el souvenir se ponían en pleito con otro, se escondían detrás de los árboles e iniciaban una escena de ficticio enfrentamiento acompañados por el arco y la flecha como auxiliares lúdicos. Para los productores y vendedores, en cambio, el arco y la flecha eran y son en la actualidad un arma de caza, de recolección de alimentos, de pesca y de intervención sobre los productos de la naturaleza. La venta de estos objetos como souvenirs de viaje se vinculaba con la 
generación de un ingreso económico extra pero su significado (en producción y en consumo) despliega una distancia muy interesante de retomar analíticamente.

En pocas palabras, el desajuste que observó la antropóloga se vinculaba con las imágenes y representaciones previas de cada sujeto en su propio contexto y trayectoria. Ahora bien, de lo que se trata, en cada uno de los casos, es de investir de significado a esos objetos y no deja de ser curiosa la manera en la que los "externos" imprimían sus propias imágenes de enfrentamiento a un elemento que, para los "locales" era una herramienta cotidiana del trabajo de recolección.

El lugar que estos "souvenirs" ocupan en la cotidianeidad de ambos grupos es menor: para unos es una posibilidad de venta y de obtención de dinero y, para otros, es un medio de "replicar" una experiencia vivida in situ. No vamos a trabajar aquí las nociones de experiencia pero sí retomaremos el concepto en el sentido que le imprime Thompson (1995): la experiencia la construyen los propios sujetos en el curso de sus vidas y se articula en las relaciones que éstos establecen con otros sujetos y los significados que los rodean. Es por ello que la compra de un souvenir (que difícilmente sea reutilizado una vez finalizado el viaje, tal es el destino de casi todos los pequeños recuerdos que se compran y venden a escala global) nos informa también del lugar que ocupan las excursiones a espacios diferentes al cotidiano y los objetos que se compran con sentido lúdico para ser utilizado en otros contextos. No es central aquí analizar qué ocurre con esos objetos sino que nos pareció relevante dar cuenta del encuentro de dos conjuntos de significados diferentes que se organizan en función de percepciones, tensiones y representaciones que se explican solamente por los entramados sociales, culturales y económicos sobre los cuales se construyen.

Es por ello que no es tan importante qué juego electrónico o qué soldado de plomo o plástico se utilice ni de qué formas: de lo que se trata es de identificar las tramas en las que el campo semántico de la guerra, de las armas y del enfrentamiento se reactualiza en base a diferentes soportes, dispositivos y objetos de juego. Y es esa variedad la que le imprime no sólo la actualidad a dicho campo sino que lo reactualiza con cada juego, objeto o souvenir que aparece en los mercados más o menos formales que se utilice como juguete o con un sentido lúdico. La muerte, la guerra y las armas son tres dimensiones presentes desde siempre en los juegos pero especialmente en las representaciones y en las tramas sociales que organizan la percepción de objetos (no necesariamente connotados con la guerra y las tensiones).

Para finalizar con esta dimensión es necesario que identifiquemos un proceso complementario al mencionado: la relación entre el cuerpo y los juegos de guerra. $\mathrm{Si}$ bien el tipo de juegos y juguetes se mantiene en diferentes formas como continuidad con el pasado en sus temas, dinámicas y tensiones, hay un nuevo universo que articula el cuerpo de los más chicos, las armas y los enfrentamientos de guerra o entre "bandas". Nos estamos refiriendo a los juegos de consolas con control de movimiento que modifican la relación que los jugadores tenían con la computadora: no se trata ya de un control que se dirige con las manos sino de un sistema que "lee" los movimientos corporales que son necesarios para jugar los juegos. Ante las extendidas críticas por el sedentarismo que alentaban los juegos en línea, la "respuesta" del mercado fueron las consolas de juego con controles de movimiento que exigían a los jugadores series de movimientos corporales para activar las dinámicas lúdicas en las pantallas (CABELLO, 2009 y LEVIS, 2009). Moverse para que ese movimiento se replique en la pantalla no 
sólo es una inmensa innovación tecnológica sino una manera de involucrar el cuerpo en los juegos entre pantallas.

La "participación" del cuerpo abre un nuevo panorama polémico: si analizamos los juegos y los movimientos corporales necesarios, lo que notamos en una primera aproximación es que los cuerpos de los jugadores se involucran activamente en el acto de asesinar a un enemigo imaginario (GRANER RAY, 2004 y JENKINS, 2000). Es decir, jugar a estos juegos incluye activar gatillos de artefactos "reales" que tienen un correlato en la pantalla. . Jugar un juego de guerra o de enfrentamiento entre las pantallas incluye, en la actualidad, involucrarse con el cuerpo para ejecutar las acciones que el juego demanda en un orden preciso y con un repertorio limitado de movimientos posibles para que el dispositivo pueda interpretarlos adecuadamente.

En síntesis, no sólo encontramos una extensión de los alcances de los objetos del campo semántico de la guerra sino una presencia temática que se potencia por las nuevas dinámicas de juego que proponen las consolas de juego con control de movimiento. Involucrar el cuerpo en la ejecución y en la muerte de un enemigo en pantalla puede ser visto, a la vez, como un "avance" respecto de las críticas sobre el sedentarismo y como una exacerbación de la violencia en los juegos. El campo semántico se amplia, extiende y complejiza y la presencia de las armas más o menos sofisticadas se naturaliza en las pantallas como si ellas fueras una parte fundamental de los juegos disponibles en la actualidad.

\section{Continuidades, transformaciones y nuevas indagaciones}

El recorrido que aquí planteamos se organizó en torno a dos ejemplos sintetizadores con algunos ejemplos ilustrativos. Por un lado, el dominó como juego "residual" nos permitió profundizar las diferentes apropiaciones que, desde la escuela hasta el mercado de bienes, se realizaron de este juego. Dijimos que no se trataba de una modificación de las dinámicas de juego sino de los personajes, los números y las combinaciones posibles en cada una de las versiones del juego. Princesas, superhéroes y números aparecían dentro del universo de los juegos de los niños y de las niñas pero con sentidos diversos. No se modifican los juegos pero sí los universos que en ellos se representan. La inclusión de personajes de la televisión opera, en gran parte, como una continuidad de los entramados de consumo que los medios de comunicación contemporáneos proponen (DUEK, 2014). Y es por esta dimensión problemática y la noción de entramados de consumo que el dominó ha sido, a nuestro juicio, una productiva manera de ilustrar el recorrido de un juego considerado "tradicional" a la luz de los contextos en los que se desarrolla, crece y se modifica.

El segundo ejemplo, el de los juegos de guerra o de enfrentamientos también nos demandó una revisión del lugar de los soldaditos de plomo en la historia y del recorrido que los juegos organizados en torno de estas temáticas han tenido a lo largo del tiempo. La relación de los más chicos con objetos rígidos, fríos e inalterables llega a modificarse a tal punto que hoy vemos de qué modos los movimientos del cuerpo son necesarios para "ejecutar" las acciones deseadas en las pantallas.

No es nuestro objetivo marcar pasados arcádicos ni tampoco presentes funestos: nuestro objetivo aquí radica en la exposición de parte de las conclusiones de nuestro trabajo que se orientan a la identificación de grandes continuidades más que de transformaciones. Los objetos del juego parecen cambiar pero, en un análisis en 
profundidad, encontramos continuidades de temas, de dinámicas y de expectativas por parte de los juegos disponibles. La relación de los niños y niñas con los entramados de consumo y el reconocimiento permanente de los personajes, propuestas e interacciones nos permite abrir un universo problemático en el que debemos indagar sobre los efectos, las materialidades y los objetivos de los juegos en el contexto en el que se desarrollan en la actualidad.

Por otra parte, deviene central mencionar que los nuevos dispositivos y las nuevas tecnologías aplicadas a los juegos han modificado algunas dimensiones del universo lúdico. Mencionamos, por ejemplo, la relación de los cuerpos de los jugadores con los movimientos de las pantallas y el sentido que tiene (y esto es nuevo por la conexión del cuerpo y la pantalla a través de un sensor de movimiento) la conversión del jugador en ejecutor de las acciones del juego. Ahora bien, salvo esta excepción no menor, lo que identificamos es que las temáticas de juego, los personajes y las dinámicas no se han modificado sustancialmente. Las tramas y las historias que se construyen en los juegos hablan más de continuidades históricas que de transformaciones sustantivas del universo lúdico infantil.

Es por ello que, en un campo de estudios novedoso pero extendido, consideramos interesante la afirmación sobre las continuidades en los juegos. Kafai (2000) sostiene que ha habido una transformación de los diseños de juegos electrónicos a la luz de las diferencias de género identificables en la actualidad. A su vez, Turkle (2012) identifica la noción de "alone together" que implica la relación que se establece entre los universos lúdicos infantiles contemporáneos en términos de individualidades organizadas en función de las pantallas como "factores de unión".

El potencial educativo de los nuevos juegos electrónicos y de los tradicionales ha sido identificado por Jenkins y Cassell (2000) en diferentes abordajes de un libro central para pensar los juegos contemporáneos: From Barbie to Mortal Kombat que tuvo su secuela en Beyond Barbie and Mortal Kombat (KAFAI et al., 2008). En ambos libros, que son parte de una "saga", se abordan cuestiones vinculadas a los juegos electrónicos, al género y a las transformaciones que las nuevas tecnologías han supuesto para los niños y niñas que juegan.

Este trabajo que aquí estamos finalizando se propone ubicarse en los márgenes de ambos libros pero retomando sus preocupaciones respecto del rol social de los juegos, de sus transformaciones, de sus posibilidades tecnológicas pero también de sus dimensiones narrativas, de la construcción de tramas y de significados. Identificar las apropiaciones mercantiles, escolares y sociales del dominó nos permitió recorrer la historia de los juegos y las formas en las que los contextos en los que se desarrollan intervienen y modifican las prácticas. Lo mismo ocurre en el análisis de los juegos de guerra y la extensión del campo semántico que allí se abre.

En síntesis, estamos proponiendo retomar algunas consideraciones de Williams (1988) que ubicaban a las prácticas culturales no sólo entramadas en la historia sino en los condicionamientos políticos, económicos y educativos de los sujetos que participan en ella. El análisis cultural es una puerta de entrada a los universos de las decisiones, de los objetos, de las tensiones y de las potencialidades que la vida cotidiana abre para los sujetos que la transitan. Consideramos, entonces, que este análisis que aquí presentamos, permite orientar la mirada hacia los juegos como permanencias históricas pero, complementariamente, como permeables a los contextos en los que se desarrolla. 
Los juegos y sus materialidades son accesos privilegiados para el análisis cultural porque que abren, orientan, construyen significados y clausuran universos sólo a través de los sujetos que juegan y se sumergen, por un rato, en universos ajenos en los que devienen, de diferentes formas, protagonistas.

\section{Bibliografía}

BENJAMIN, W. Infancia en Berlín. Buenos Aires: Alfaguara, 1990.

BROUGÈRE, G. Jogo e educação. Porto Alegre: Artes Médicas, 1998.

BROUGÈRE, G. El niño en la cultura lúdica. In: Revista Lúdicamente, Nº4, Buenos Aires, 2013.

CABELLO, R. Las redes del juego. Buenos Aires: UNGS, Prometeo, 2008.

CABELLO, R. (org.). Ciberjuegos. Escritos sobre usos y representaciones de los juegos en red. Buenos Aires: UNGS, Imago Mundi, 2009.

CAILLOIS, R. Los juegos y los hombres. La máscara y el vértigo. México: FCE, 1964.

CANTORA, A. y Molinari, L. Jugar a "Mundo Gaturro": Acerca del juego, la exhibición y el consumo en la plataforma online. Tesina de Grado en Ciencias de la Comunicación. Buenos Aires: UBA, 2012.

CASSELL, J. y JENKINS, H. Chess for girls? Feminism and computer games. In: From Barbie to mortal kombat: Gender and computer games. Boston: MIT Press, 2000.

DUEK, C. El juego y los medios: Autitos, muñecas, televisión y consolas. Buenos Aires: Prometeo Libros, 2012.

DUEK, C. Infancias entre pantallas: Las nuevas tecnologías y los chicos. Buenos Aires: Capital Intelectual, 2013.

DUEK. C. Juegos, juguetes y nuevas tecnologías. Buenos Aires: Capital Intelectual, 2014.

ENRIZ, N. Jeroky Porã, juegos saberes y experiencias infantiles Mbyá guaraní en Misiones. Muenchen: Lincom Europa, 2011.

ENRIZ, N. Neovanga. Juegos infantiles Mbyá guaraní en Misiones. Saarbrücken: EAE, 2012.

GARCÍA GALERA, M. Televisión, violencia e infancia. Barcelona: Gedisa, 2000.

GLANZER, M. El juego en la niñez: Un estudio de la cultura lúdica infantil. Buenos Aires: Aique, 2000.

GUBER, R. De chicos a veteranos, memórias argentinas de La guerra de Malvinas. Buenos Aires: Antropofagia, 2004.

HOGGART, R. La cultura obrera en la sociedad de masas. México: Grijalbo, 1990.

HUIZINGA, J. Homo Ludens. Buenos Aires: Emecé. 1939.

JENKINS, H. Complete freedom of movement: video games as genderes play spaces. In: CASSELL - JENKINS (org.) From Barbie to mortal kombat. Boston: MIT Press, 2000.

KAFAI, Y. Video game designs by girls and boys: variability and consistency of gender differences. In: CASSELL - JENKINS (org.) From Barbie to mortal kombat. Boston: MIT Press, 2000. 
KAFAI, Y. Gender play in a tween gaming club. In: KAFAI, Y; HEETER, C. DENNER, J; SUN, J. (org.). Beyond Barbie and mortal kombat. Boston : MIT Press, 2008.

KAFAI, Y; HEETER, C. DENNER, J; SUN, J. (org.). Beyond Barbie and mortal kombat. Boston : MIT Press, 2008.

LEVIS, D. Videojuegos en red: espacios simbólicos de juego y encuentro. In: APARICI, R. (org.). Comunicación educativa en la sociedad de la información. Madrid: Universidad Nacional de Educación a Distancia, 2003.

MANSON, M. et DE LA VILLE, I. Los fabricantes de juguetes y juegos franceses y su responsabilidad social respecto del niño (1891-1911). In: Revista Lúdicamente, ํo4, Buenos Aires, 2013.

NUNES, A. Entre juegos y tareas: Una etnografía de las actividades cotidianas de los niños A’uwë-Xavante (MT, Brasil). In: Revista Lúdicamente, №4, Buenos Aires, 2013.

PLATH, O. Aproximación histórica-folklórica de los juegos en Chile. Santiago: Nascimento, 1986.

PELEGRINELLI, D. Diccionario de juguetes argentinos: Infancia, industria y educación 1880-1965. Buenos Aires: El juguete ilustrado, 2010.

RODRÍGUEZ TAPANÉS, B. y HERNANDEZ DE LARA, O. Pasatiempos en la vida militar. Juegos y juguetes en el castillo de San Severino, Matanzas, Cuba. In: Revista Cuba Arqueológica, año 1, nº 1 , Cuba, 2008.

ROSSI CARDOSO, S. Memórias e jogos tradicionais infantis: lembrar e brincar é só começar. Londrina: Editora da Universidade Estadual de Londrina, 2001.

SCHEINES, G. (org.). Los juegos de la vida cotidiana. Buenos Aires: Eudeba, 1985.

SUTTON SMITH, B. The Measurement of Masculinity and Femininity in Children. In: ROSENBERG, B. G.; SUTTON-SMITH SOURCE, B. Child Development. Vol. 30, No. 3 (Sep., 1959). Londres: Blackwell, 1959.

SUTTON SMITH, B. The Expressive Profile. In: The Journal of American Folklore. Vol. 84, No. 331, Toward New Perspectives in Folklore (Jan. - Mar., 1971). American Folklore Society, 1971.

SUTTON SMITH, B. The Useless Made Useful: Play as Variability Training. In: The School Review. Vol. 83, No. 2 (Feb., 1975). Chicago: The University of Chicago Press, 1975.

THOMPSON, E. Cuestiones en común. Barcelona: Crítica, 1995.

TURKLE, S. Alone together: Why we expect more fron technology and less from each other. New York: Basic Books, 2012

WILLIAMS, R. Marxismo y literatura. Barcelona: Península/Biblos, 1988. 